\title{
ENCADENAMIENTOS Y SIMILITUDES ESTRUCTURALES PARA LAS REGIONES DE CHILE
}

\author{
LINKAGES AND STRUCTURAL SIMILARITIES FOR THE REGIONS \\ OF CHILE
}

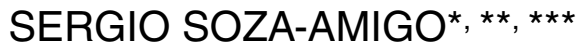

Universidad de Magallanes

\begin{abstract}
Many authors stress the importance of relationships between sectors for the economic growth. Therefore the concept of linkage appears in the definition of two of the most traditional concepts in the input-output analysis: the "key sector" and cluster.

In this paper, key products and activities are identified for the Chilean regions, the clusters that form and the structural similarity that exists between them. For this, we use a combination of so-called Important Coefficients and Fields of Influence.

The results indicate that the regions that are most similar are: Tarapacá and Antofagasta, Biobio and Los Lagos. Besides the "developed" regions concept that is associated with a high division of labor and coordination between sectors, they have similar production structures and are very different from those that have "less development".
\end{abstract}

Keywords: Structural analysis, sensibility analysis, important coefficients, fields of influence.

JEL Classification: C67, D57, R15, R34 and Z00.

* Facultad de Ciencias Económicas y Jurídicas, Av. Bulnes 01855, Punta Arenas, Chile. E-mail: sergio. soza@umag.cl.

** Se reconoce y agradece el apoyo del FONDECY, proyecto número 11070168: "Análisis Estructural de la Economía Magallánica desde la Perspectiva del Enfoque Input-Output" y del Núcleo de la Iniciativa Científica Milenio "Ciencia Regional y Políticas Públicas".

*** El autor desea agradecer los comentarios y sugerencias de los árbitros anónimos. Cualquier error u omisión es responsabilidad del autor, no comprometiendo por tanto a los anteriores, a esta revista, ni a la Universidad de Magallanes. 


\section{Resumen}

Muchos autores destacan la importancia de las relaciones entre sectores para el crecimiento económico. Por ello, el concepto de linkage aparece en la definición de dos de los conceptos de mayor tradición en el análisis input-output: el sector clave -key sector-y el aglomerado o clúster.

En este trabajo se identifican los productos y actividades que son claves para las regiones chilenas, los clúster que forman y la similitud estructural que se da entre estas. Para ello se utiliza una combinación de los denominados Coeficientes Importantes y los Campos de Influencia.

Los resultados indican que las regiones que más se parecen son: Tarapacá con Antofagasta y Biobío con Los Lagos. Además, las regiones "desarrolladas", concepto que se asocia a una alta división del trabajo y articulación entre sectores, presentan estructuras productivas similares y muy disímiles de las que acusarían "menor desarrollo".

Palabras Clave: Análisis estructural, análisis de sensibilidad, coeficientes importantes, campos de influencia.

Clasificación JEL: C67, D57, R15, R34 y Z00.

\section{INTRODUCCION}

Esta investigación busca entregar información sobre las subestructuras económicas que existen en Chile, limitadas y acotadas por la definición geopolítica de las regiones que forman el país. Las respuestas se centran en el entramado económico y en las similitudes estructurales que ellas presentan. La estructura se obtiene a partir de los Campos de Influencia (CdI) que forman los distintos coeficientes técnicos de cada región, obteniéndose a partir de tal procedimiento los key sector. En forma adicional, y a modo de estudio complementario, se realiza un Análisis del Tipo Causal (triangulación de matrices a partir de sus funciones de demanda), con el fin de identificar los bloques no descomponibles o clúster de cada región.

Comparar regiones es importante pues facilita que aquellas relativamente atrasadas puedan seguir el curso de las desarrolladas. Además, por medio de la observación del conjunto de regiones y en lo particular del vínculo que forman las distintas actividades, se puede analizar si entre ellas existen características comunes. Así, y en función de estas peculiaridades, sería posible traspasar el desarrollo tecnológico a las regiones que las secundan. En definitiva, las exitosas actuarían como verdaderos spillovers al traspasar su desarrollo tecnológico a las atrasadas. En base a lo expuesto, se plantean las siguientes hipótesis: 
H0: Las regiones desarrolladas presentan CdI y PIB per cápita, superiores a la media.

$\mathrm{H} 1$ : Las regiones que presentan unos CdI superiores a la media tienen una estructura productiva similar entre sí.

$\mathrm{H} 2$ : Las regiones desarrolladas versus las no desarrolladas presentan estructuras económicas no homogéneas, y éstas varían de acuerdo a sus procesos productivos (funciones de producción y distribución).

H3: Un estudio estructural input-output para las regiones de Chile facilita la interpretación de sus estructuras.

Dado lo anterior, el objetivo central de este estudio es profundizar en aspectos relacionados con el análisis estructural input-output (io). En este orden, se plantea un análisis que posibilite un conocimiento y comprensión de las economías objeto de estudio y observar qué regiones de Chile presentan un entramado económico similar desde la perspectiva de los CdI que forma y de los bloques no descomponibles con que cuenta (clúster). De igual modo y, basados en sus CdI, se detecta cuáles son más o menos desarrolladas en la medida en que éstas presenten unos campos más vinculados entre sí y posean a su vez, una cantidad tal de ellos que los posicione por sobre la media.

Por otra parte, este estudio permite - de contar con nuevas matrices-contrastar las hipótesis planteadas a posteriori y observar cuál ha sido la evolución de cada economía, en términos individuales y grupales, indagando cómo han variado sus CdI (¿son más o menos profusos en el futuro?, etc.) y cómo tal variación las posiciona respecto a las otras regiones en términos de desarrollo, esto es, responder: ¿presentan un nivel de entramado en términos de profusión (entrelazado de actividades) mayor a la media?, ¿aumentaron o disminuyeron sus CdI?, ¿son más o menos profusos? En tal sentido, la metodología es fácilmente replicable en la medida que las nuevas matrices sean coherentes en cuanto a la obtención y tamaño con las antiguas. Teniendo cubiertos estos aspectos, el paso siguiente sería analizar las evoluciones en las distintas economías, poniendo especial cuidado en la división del trabajo y vínculos que forman las actividades económicas (productos y servicios), lo que se puede contrastar simultáneamente con la evolución del PIB per cápita de cada región.

Estudiar las subestructuras que forman la economía de Chile ayuda a entender los funcionamientos de las economías regionales y las semejanzas que en estas se presentan, lo que facilita el comprender no sólo cuál será el posible impacto de una determinada política económica nacional, sino que cómo y a cuántas regiones puede afectar. Además este análisis permite saber qué áreas potenciar al interior de cada región, aspecto importante si se considera que las economías están en constantes fluctuaciones. Aun cuando el estudio se refiere a una época concreta, ello no quita que sus respuestas a futuro puedan incorporar aspectos dinámicos haciendo las consideraciones pertinentes.

Para determinar la importancia de un producto o actividad se considera la génesis y desarrollo del concepto "rama clave" que fuera planteado por Hirschman en 1958, inducido a nuestro modo de ver por el trabajo de Rasmussen de 1956; posteriormente, 
sobre la base de la Técnica de los Límites Tolerables, se confeccionan para cada región los CdI de cada uno de sus productos o actividades. Definidos éstos, se identifican cuáles son claves, conjugando la mencionada técnica con el significado tradicional del concepto. Luego, utilizando un clúster jerárquico, se determina qué regiones guardan un cierto parecido desde la perspectiva de las actividades más sensibles. Por último, se triangulan las distintas matrices con el fin de detectar cuáles son los productos o actividades que forman lo que se denomina "el grupo no descomponible" para cada región, idea que se asemeja al concepto de clúster, pues para que el mismo exista depende de lo que ocurra con las otras actividades que componen el grupo.

Los resultados son interesantes y muestran que la identificación de los productos y actividades claves y similitudes estructurales que se presentan en y entre algunas regiones son consistentes con lo observado; por ejemplo, se detecta la evidente importancia que tiene el sector alimentos y frutas en el centro sur del país. Este aspecto es relevante ya que a partir de tales semejanzas se puede sugerir tomar como base el diseño de programas de desarrollo productivo de regiones exitosas en determinadas actividades. A modo de ejercicio de benchmarking, se puede proponer imitar a las regiones líderes, basados en las similitudes detectadas en las estructuras productivas y en los productos o actividades claves; lo anterior basado en que la información procesada da suficiente evidencia como para apoyar procesos de desarrollo productivo en regiones menos aventajadas o con miras a potenciar ciertas actividades que no le son propias.

Con respecto al instrumento empleado, el análisis input-output (a-io) considera un entorno multisectorial que permite un estudio estructural basado en un conjunto de productores que demandan y ofrecen insumos con el fin de efectuar su actividad. Se asume que la tecnología es constante y permite el máximo beneficio. Cada bien o servicio que se elabora se hace bajo la premisa de que se combinan proporcionalmente los mismos bienes, servicios e inputs primarios (tecnología de la actividad). En este orden, cada actividad se puede agrupar de acuerdo a lo que produce y la tecnología empleada; en este sentido, la técnica responde al objetivo trazado. Un análisis que facilite el comparar estructuras productivas, pues ellos son fruto de la interpretación, al decir de Pulido y Fontela (1993), "de un instrumento básico de la coherencia de datos de contabilidad regional entre las diferentes ópticas posibles de la valoración del producto (consumos intermedios, valores añadidos, rentas y demanda final)".

Dado que las tablas utilizadas están referidas al mismo año y siendo coincidentes con Aroche (2006), se omite la existencia de cambios estructurales motivados por tecnología, o bien producto de causas tales como crecimiento sectorial, acumulación de capital; o, en especial, producto de la forma que adoptan las relaciones intersectoriales. En este mismo orden, la metodología tiene por desventaja el no vincular explícitamente (aunque sí lo hace implícitamente en la demanda final) el cruce de información entre bienes públicos y sectores; este es un aspecto que a futuro se puede abordar incorporando, por ejemplo, un vector que exprese una relación entre transporte y producción. 


\section{HACIA UNA DEFINICION DE PRODUCTO O ACTIVIDAD CLAVE Y DESARROLLO}

Existe cierto consenso sobre la importancia entre la relación linkage y desarrollo en un entorno io, así como en que este proceso es impulsado por un número escaso de actividades, las que serían del tipo "clave". Sin embargo, la definición y estimación de lo que se denominan sectores, productos o actividades claves, aún está sometido a discusión. La literatura se ha centrado en rebatir las diferentes concepciones o definiciones de lo que es o debe ser un key sector. Se ha intentado mostrar la superioridad de algunas técnicas frente a otras, pero sin llegar a un acuerdo total.

De igual modo, existe una serie de planteamientos que llevan a resolver qué rama, sector o actividad económica es más importante, en el sentido de lo que representa bajo un determinado prisma y la técnica que se utiliza para evaluarlo. Sin embargo, en la literatura no se establece qué procedimiento es mejor o cuál es más eficiente para ello. Soza-Amigo (2007) plantea que no existe un enfoque y, por añadidura, técnica que sea superior a otra. Los distintos enfoques que determinan la relevancia de un sector para una economía no son sustitutos entre sí, sino complementarios; luego, entre más visiones se conjuguen para detectarlo, mejor será, en pro de evaluar la relevancia de una rama o actividad para el sistema productivo que se analiza, puesto que más acertada será su categorización en el contexto que se establezca.

Por otra parte, se observa que existe un vínculo entre los linkage, el desarrollo y la estructura de las economías. En este sentido Leontief en 1963 indicaba bajo un contexto io que "cuanto más desarrollada está una economía, más se parece en su estructura interna a la de otras economías desarrolladas", lo que a nuestro entender se podría interpretar, bajo ciertas condiciones, como una suerte de "principio de igualdad en las estructuras de las economías que son desarrolladas". También señalaba que la estructura interna de cada economía es un aspecto que tiene que ver con los tipos de relaciones, agregando que, mientras más desarrollada es ella, más complejo se hace su vertebrado económico, es decir, si una economía manifiesta poseer respecto a otra un alto número de ramas o actividades que están encadenadas entre sí -alta y variada dependencia de oferta y demanda- se aceptará, bajo la condición anterior, que es más desarrollada.

Complementando la idea de Leontief referida al desarrollo en un entorno io, en Miller y Blair (1985 y 2009) se hace mención especial al trabajo de Carter de 1970, en donde se destacaba que "cuando una economía se desarrolla, la división del trabajo entre las industrias se despliega, se dispersa, esto es, se expande y a su vez se torna más compleja", lo que refuerza las ideas de desarrollo basadas en la complejidad y similitud estructural planteadas por Leontief.

Con argumentos similares, Forsell en 1988 sostenía que "a medida que más compleja se hace una economía, su número de coeficientes nulos tiende a decrecer y, en contraposición, sus coeficientes más importantes tienden al incremento", es decir, a medida que se desarrolla una economía su sistema se hace más integrado, a lo que se puede agregar que aumenta la interdependencia entre actividades.

En una línea similar y más contemporánea, se argumenta que "a medida que se desarrolla una economía la cantidad de relaciones indirectas que posee e interacción 
con otros sectores aumenta. Además, tales relaciones implican un número creciente de ramas" (Aroche, 1996). Adicionalmente, Cardero y Aroche (2008) plantean que, "para el caso de Corea del Sur y Noruega, se observa que el paso de una estrategia sustitutiva de importaciones por una de exportaciones no implicó una desintegración de sus aparatos productivos internos". En tal sentido, con estas dos ideas se confirma lo sostenido por Leontief en 1963 y por Carter en 1970 respecto a la división del trabajo en las economías desarrolladas y que el vertebrado económico no se vería afectado cuando aumenta considerablemente la producción, sino que, por el contrario, facilita el aumento de las relaciones de intercambio dada la especialización que se va logrando.

Con respecto al último punto, se puede agregar lo sostenido por Imbs y Wacziarg (2003), "mientras una economía se desarrolla pasa por diversos umbrales de especialización". En tal sentido se confirma que en la medida que se inicia tal proceso se pasa por variados tipos de especialización y se van creando más diversificaciones productivas y laborales, luego el entramado de la economía se torna más complejo. ${ }^{1}$

En consonancia a lo manifestado, se puede defender la idea de que en un entorno io una economía será considerada más desarrollada respecto a otra si presenta una estructura económica con una alta división de actividades, esto es, se muestra muy profusa en términos de intercambio respecto a la economía con que se compare.

En base a lo expuesto, la experiencia recogida en $2008^{2}$ y dado que el planteamiento de los CdI obtenidos a partir de la técnica de los límites tolerables muestra la interrelación de un producto o actividad con el resto de la economía y permite ahondar en la cuantía de las etapas productivas más importantes, así como el valor en que ellas deben cambiar, a objeto de modificar la producción en, por ejemplo, un 1\%, se considera oportuno detectar cuáles son, bajo esta premisa, los CdI que se forman en las regiones de Chile. Por otra parte, se cree pertinente identificar bajo la condición anterior qué regiones guardan entre sí un cierto parecido, así como revelar en cuáles se observa la presencia de algún clúster (bloques no descomponibles). Lo planteado se realiza considerando que la economía es abierta, es decir, se asume que las distintas rentas no se gastan necesariamente en cada región.

\section{CAMPOS DE INFLUENCIA (CdI)}

Dada la variedad de enfoques y técnicas que permiten identificar lo que se entiende son los key sector y en consideración a que de estos se espera que a lo menos incrementen la producción por los vínculos que ellos presentan con otras ramas, sectores, productos o actividades, afectando finalmente los niveles de renta, en este trabajo se ha considerado oportuno el identificar estos key sector utilizando

1 En un sentido práctico, comprender tal proceso puede servir para corroborar en qué se ha especializado una economía para una data en particular, lo que puede ayudar a develar el futuro en términos de la especialización que vendrá, o bien, ayudar a encauzarla.

2 Nos referimos al trabajo "Análisis comparativo para la economía magallánica desde la perspectiva del enfoque input-output”, Revista de Análisis Económico, 23 (2): 95-120, 2008. 
la técnica de los CdI. Hewings et al. (1989) plantean evaluar simultáneamente el tipo de vínculo que existe entre ellas y su nivel de sensibilidad medido en términos de producción.

El enfoque identifica el impacto de la alteración de un coeficiente $a_{i j}$ sobre aquellos que forman la matriz inversa ( $a_{i j} \in A ; A=$ matriz de coeficientes técnicos). La metodología en sí se puede resumir de la siguiente manera: se toma un coeficiente $a_{i j}$ y se observa el efecto que produce su variación sobre el sistema matricial. Si existe un elemento que destaque del resto y que sea importante para este coeficiente, pasará a formar parte de lo que se denomina su CdI. Tal proceso permite graficar la complejidad de una estructura económica en base a los pares de coeficientes que se representan. Si dicha representación es muy entrelazada, indicará que sus interrelaciones están muy desagregadas y, por tanto, cada producto o servicio que forma parte del campo tendrá un alto impacto sobre el sistema productivo. La situación inversa mostrará la existencia de acciones altamente concentradas o que la economía depende de muy pocas actividades, las que probablemente serían independientes.

A partir del criterio anterior, se puede realizar una jerarquización entre los coeficientes y, por extensión, entre los productos o actividades en función de que contengan o no un número destacado de estos coeficientes. Si fuese el caso, se trataría de una actividad muy sensible o clave, ya que sus cambios conllevarían importantes impactos en la economía.

El trabajo de Hewings et al. (1989) aporta la idea de complementariedad entre una técnica que evalúa qué actividades son claves con otra que determina qué coeficientes son más sensibles ante un cambio en la economía. En este sentido, emplea un principio similar al que presentan Schintke y Stäglin en 1988. Sin embargo, lo que Hewings et al. (1989) plantean se refiere a una técnica que permite representar gráficamente las interrelaciones más importantes entre dos coeficientes, facilitando evaluar cuán compleja puede ser una economía, desde el punto de vista de poseer más o menos actividades interrelacionadas, sumado al principio anterior. En este sentido, se puede aceptar que bajo el contexto io, la tradición indica que es posible asumir que una economía es más desarrollada respecto a las que se compara si cumple con el requisito de presentar una alta división de actividades que se vinculan mutuamente, esto es, que presente unos CdI profusos respecto a la economía con que se coteje.

\section{METODOLOGIA}

Dada la similitud que se da entre las propuestas de West (1982) y Hewings et al. (1989), tanto en sus planteamientos como en sus formulaciones, y luego de comprobar que, para estas, se pierde de vista el concepto de elasticidad desarrollada por Sebald en 1974 y, por otra parte, debido a que no conjuga la variación que se plantea con la producción total, se descarta dicho enfoque, y se opta por emplear la formulación de Schintke y Stäglin (1988) para la detección de la importancia en términos de sensibilidad de cada coeficiente. Es decir, en este trabajo se toma la idea de CdI desarrollada por Hewings et al. en 1989, y se emplea parcialmente el planteamiento presentado por 
Aroche en $1996 .{ }^{3}$ En este sentido, lo que se hace es utilizar la formulación presentada por Schintke y Stäglin en 1988, y no la de Schnabl de 1994 o la de Holub \& Schnabl de 1994, que son las usadas por Aroche en 1996, 2002 y en 2005. Lo último obedece a que estas expresiones son una reducción de la ecuación desarrollada por Schintke y Stäglin y, por tanto, representa una parcelación del fenómeno estudiado.

La fórmula presentada por Schintke y Stäglin en 1988 (ecuación 1) nace de la idea intuitiva de evaluar el efecto que se produce en la economía tras alterar ex profeso una de las distintas etapas productivas en un determinado porcentaje, el que genéricamente es conocido como "p".

$$
w_{i j}(p)=a_{i j}\left[l_{i j} p+100\left(l_{i j} / x_{i}\right) x_{j}\right]
$$

Al revisar la ecuación (1), se constata que $w_{i j}$ representará el grado de importancia que logra cada $a_{i j}$ (coeficiente técnico). Cuanto mayor sea el valor de $w_{i j}$ más significativas serán las compras intermedias que realiza la $j$-ésima actividad a la $i$-ésima, es decir, señala la importancia que tiene el coeficiente $a_{i j}$ en términos de los efectos que genera una alteración "p" en la producción; $p$ representará el porcentaje máximo de variación que se provocará sobre la producción (límite tolerable de error), siendo $l_{i j}$ un elemento de la matriz inversa de Leontief $\left[(I-A)^{-1}\right]$ y $x_{j}$ la producción del sector j-ésimo.

Además, puede definirse un $r_{i j}=1 / w_{i j}$ como el inverso de las elasticidades, donde la sensibilidad del coeficiente de producción vendría dada por este cociente, que será tanto más reducido cuanto más importante sea $a_{i j}$ (Schintke y Stäglin, 1988).4;5

Respecto al trabajo que se abordará, primero se realizará por cada matriz una sucesión de cambios, lo que dará origen a una nueva matriz por cada uno de ellos y, dado que cada matriz tiene una dimensión de $40 * 40$ entre productos y/o actividades, se obtendrá un total de 1.600 matrices por zona geográfica. De esta forma cada una de ellas representará el cambio en un elemento $a_{i j}$. A continuación se confeccionan las respectivas matrices booleanas para cada región y, a partir de estas matrices, se

3 Lo que propone Aroche tanto en 1996 como en 2002 y 2005 es una extensión del trabajo desarrollado por Hewings et al. El conjuga los planteamientos anteriores con la formulación de Schnabl de 1994 y la Teoría de Grafos, logrando establecer de un modo gráfico las relaciones de dependencia de cada actividad, idea que, como se verá más adelante, es parcialmente tomada en este trabajo.

4 Un aspecto interesante de comentar sobre este punto es el que se encuentra en Robles y Sanjuán (2008, pp. 191), donde se hace un paralelo entre los MICs (coeficientes más importantes) y la formulación presentada por Schintke y Stäglin para la determinación de $w_{i j}$. En tal artículo se ven las similitudes que se presentan luego de eliminar el autoconsumo en el modelo de demanda; si ello ocurre, se observará que $l_{i i}$, elemento de la diagonal de la inversa de Leontief, será igual o estará muy próximo a la unidad. Por otra parte, el producto $a_{i j} x_{j} / x_{i}$ tendrá como resultado los coeficientes de distribución $b_{i j}$, luego en la fórmula recogida se suman unos números muy pequeños $l_{i j} p / 100$ a los citados coeficientes. Así, se podría considerar a los MICs como unos elementos muy próximos a los $b_{i j}$ más grandes o significativos y, por tanto, "tamaño e importancia quedarían directamente relacionados. Si $b_{i j}$ es grande $-\mathrm{o} r_{i j}$ pequeño- el correspondiente $a_{i j}$ es importante".

5 Para comprender esto último, asuma que el límite tolerable planteado es $p=1 \%$. Si en estas condiciones un coeficiente $a_{i j}$ presenta un $r_{i j}=5$, éste se debe interpretar como: una variación del $5 \%$ en el coeficiente $a_{i j}$, con $a_{i j} \in A$, provocaría un $1 \%$ de variación en la producción sectorial. 
confeccionan los CdI en base a la técnica de grafos. Para cumplir con esta etapa, y sobre la base de la información derivada del entramado de las economías analizadas, se confecciona un Clúster Jerárquico a partir del cual se obtienen las principales semejanzas y divergencias para las regiones estudiadas. Finalmente, se utiliza un enfoque complementario conocido como "Triangulación de Matrices", proceso que permite jerarquizar las compras y ventas y detectar a su vez los grupos no descomponibles de cada economía, los cuales son definidos como "clústers", dada la dependencia mutua que se da para que ellos existan.

Para la determinación de los distintos bloques se establece como condición de partida el considerar sólo los coeficientes técnicos importantes (mic; $a_{i j} \geq 0,05$ ); dada esta restricción se definen grupos o subgrupos de productos y actividades autónomas. Una vez confeccionadas estas estructuras, las que por definición son equivalentes a triangular una matriz, se identifican las actividades o sectores jerarquizados superiores, inferiores y no descomponibles. Estos últimos son precisamente los que permiten identificar los clústers, pues los productos o actividades que lo integran dependen de una coexistencia mutua de ellos para su desarrollo (Fontela y Gilli, 1977).

El proceso funciona de la siguiente forma: considerando que el modelo tiene " $n$ " funciones (producción y distribución), se puede establecer una matriz de ocurrencias " $K$ con $k_{i j}$ perteneciente a $K$ ", la que acusará una estructura causal en la medida que se tenga dependencia de las actividades superiores, por tanto, sus elementos dependerán de que:

$k_{i j}=\left\{\begin{array}{cl}1, \text { si } b_{i j} \neq 0 & \text {, con } b_{i j} \text { elemento característico de la Matriz Inversa (producción } \\ 0, \text { si } b_{i j}=0 & \text { o distribución). }\end{array}\right.$

Por tanto, en una estructura jerarquizada se fijará en el primer lugar de la matriz de incidencias o de ocurrencias aquel producto o servicio que dependa "sólo de su demanda", el que sigue, por tanto, dependerá "de su propia demanda y del producto o servicio anterior". Como ya se entenderá, las secuencias que siguen parten de la base de la estructura anterior y la lógica establecida, lo que lleva a que se obtenga una matriz en una estructura recursiva tal como sigue:

$$
K=\left[\begin{array}{ccccccccc}
1 & 0 & \ldots & 0 & \ldots & 0 & \ldots & \ldots & 0 \\
1 & 1 & \ldots & 0 & \ldots & 0 & \ldots & \ldots & 0 \\
\vdots & \vdots & \ddots & 0 & \ldots & 0 & \ldots & \ldots & 0 \\
1 & 1 & 1 & 1 & \ldots & 1 & \ldots & \ldots & 0 \\
\vdots & \vdots & \vdots & \vdots & \ddots & \vdots & & & \vdots \\
1 & 1 & 1 & 1 & \ldots & 1 & \ldots & \ldots & 0 \\
1 & 1 & 1 & 1 & \ldots & \ldots & 1 & \ldots & 0 \\
\vdots & \vdots & \vdots & \vdots & \vdots & \vdots & \vdots & \ddots & \vdots \\
1 & 1 & \ldots & 1 & \ldots & 1 & 1 & \ldots & 1
\end{array}\right] \quad\left\{\begin{array}{l} 
\\
\text { Bloque Indescomponible } \\
\text { Jerarquización Superior } \\
\end{array}\right.
$$


De la matriz anterior se observan tres bloques: el primero corresponde a los productos o servicios jerarquizados en forma superior; el último representa al inferior, esto es, dependen de todos los anteriores, por lo que se estaría en presencia de una estructura absolutamente recursiva; en el centro lo que se denomina "bloque no descomponible", bloque que entronca con la idea de "clúster", este grupo es el que nos indicará cuán parecidos son los núcleos de las distintas economías y cuáles son las actividades que, para su existencia como bloque o núcleo, deben permanecer para que se dé su estructura, esto es, se identifica primero la similitud del bloque en términos globales y posteriormente la dependencia mutua que se da entre las actividades que lo forman y que son necesarias para que ellos existan, dado que el bloque de una región no necesariamente debe coincidir en su totalidad con el de otra, pero sí en lo que se puede denominar su "estructura vital".

\section{APLICACION Y RESULTADOS}

Para cumplir con lo planteado se emplean las matrices publicadas por MIDEPLAN, ${ }^{6}$ tomando como criterio el aceptar como límite tolerable un error de un $1 \%(\mathrm{p}=1 \%)$ y unos valores para los $r_{i j}$ menores e igual a un $15 \%$. Por otra parte, en base a lo presentado, los resultados obtenidos se pueden analizar desde al menos las siguientes perspectivas:

1. Basados en los CdI que se forman, establecer un criterio que permita la identificación de la importancia que cada producto o actividad presenta, siguiendo el esquema tradicional en función de las relaciones que ellas posean de oferta y demanda de insumos derivadas de los CdI que forma (claves -alta oferta y demanda-, impulsoras -alta demanda y baja oferta-, base -poca demanda y alta oferta-; o, finalmente, enclaves o islas, actividades que no se relacionan con el resto).

2. En base a los enlaces que se forman al interior de cada región, los resultados mostrarán cuáles son similares, en términos de la estructura que presenten. En este sentido, se responde a cuáles son las regiones que más se asemejan desde la perspectiva de los entramados que forman sus CdI, ayudando a establecer aquellas actividades en las cuales se puede planear la idea de formar grupos de asociación basados en sus procesos productivos, esto en función de sus semejanzas estructurales y la cercanía geográfica que se dé entre ellas.

3. Con el fin de jerarquizar y comparar el nivel de desarrollo se establecen dos condiciones: que la economía sea profusa en términos de los vínculos que se forman en su interior, esto es, un número de arcos superior a la media y que su PIB per cápita esté sobre la media del mismo.

6 MIDEPLAN. Aproximación a las Economías Regionales. Santiago, Chile, Ministerio de Planificación, 2005, p. 211. En este texto además se encuentra un detalle de cómo ellas fueron elaboradas, su calidad y problemas que presentan. 
4. Con un análisis adicional del tipo causal (triangulación de matrices), se puede indagar sobre cuáles son los clústers que forman los distintos productos o actividades económicas (bloques no descomponibles). Para ello y en sintonía con la tradición en el análisis io, se han considerado como coeficientes técnicos importantes aquellos mayores a 0,05 (Morillas, 2005).

\section{PRIMERAS IMPRESIONES}

Llama la atención que la Región Metropolitana no muestra un entrelazado importante. Su economía no es profusa como las de Coquimbo, Valparaíso u O’Higgins. Esto indica que la hipótesis “... entre más entrelazadas estén las ramas, más desarrolladas serán las economías...”, en tal caso no se daría. ${ }^{7}$ Una razón podría ser que las principales actividades económicas que se realizan en esta región o las que dependen de ella se realizan en áreas aledañas. Otra interpretación posible es que el aumento de su producción está asociado a la modificación de actividades concretas, no contando, por tanto, con un abanico de éstas.

Aun cuando no es prioritario analizar el fenómeno anterior, se debe tener presente que la técnica elegida no se utiliza porque explique ello a cabalidad, sino que es empleada por revelar la forma de una estructura económica, esto es, porque logra interpretarla basados en funciones de producción y distribución. Sin perjuicio de lo comentado, se puede asociar tal entramado a distintos aspectos tales como externalidades marshallianas y spillovers de conocimiento. A las primeras, dado que la concentración geográfica y especialización de determinadas actividades puede obedecer a la existencia de una mano de obra calificada y no a una fuerte y variada relación entre oferta y demanda de insumos. Esto es, aun cuando se aprecia que esta última relación existe, ella puede motivarse dada una especialización de productos o actividades cuyo entramado es poco desarrollado. Esto podría explicar por qué no ocurre lo que indica Carter en 1970 o bien Forsell en 1988, es decir, a medida que se desarrolla una economía, su sistema se hace más integrado, o bien aumenta la interdependencia entre sectores. Si existe un aumento de la especialidad en las industrias, cada una de éstas cubrirá un segmento menor del proceso productivo, lo que induce a que se incremente el número de transacciones interindustriales (Aroche, 2006, y Cardero y Aroche, 2008).

7 En consideración al entorno io en que se trabaja y dados los comentarios vertidos por Leontief (1963), Carter (1970), Forsell (1988), Aroche (1996) o Cardero y Aroche (2008) sobre las particularidades del desarrollo. En este artículo se acepta que una economía -región- será desarrollada si presenta una cantidad igual o superior a 35 arcos (excluyendo su autoconsumo) y no aquellos que están por sobre la media. Tal valor es motivado por las 4 regiones que más de éstos presentan (Atacama (35); O’Higgins (42); Valparaíso y Los Lagos (38)) y con el fin de establecer una mejor comparación se cree que tal criterio es pertinente -aunque restrictivo-dado que es un número de arcos mayor a la media y corresponde a un tercio del total de regiones; sin perjuicio de ello, tal decisión permite una mejor equivalencia del nivel de profusión, la que es equivalente a una alta división de la economía, por lo que representaría más fácilmente el concepto de desarrollo en este contexto. 
A lo anterior se debe agregar el beneficio en términos de intercambio de información que logran las direcciones de actividades por encontrarse entre empresas que realizan una misma producción (spillovers tecnológicos y de conocimiento), esto es, si bien es cierto se puede aceptar que la región es desarrollada, el modelo elegido no explica para este caso en particular el por qué de ello, pero sí indica los tipos de intercambio que en ella se dan, los que acusarían una economía menos desarrollada.

Otra razón que explique el escaso entrelazado puede ser la existencia de economías de aglomeración, la cual induciría a una especialización poco variada en términos de actividad. Aun cuando se aprecia que existe una infraestructura que permite una reducción de costos, un flujo de capitales importante y la concentración de establecimientos industriales y de servicios, este argumento revelaría parte del desarrollo metropolitano, pero cae en el contrasentido de que no explica el por qué no existe un entramado diverso y profuso, propio de las economías desarrolladas donde existen economías de aglomeración. Finalmente, con respecto a la situación de la Región Metropolitana, se hace notar que no pertenece al grupo de las regiones más desarrolladas "aquí establecidas como tal", producto del criterio fijado (número de arcos (sobre 35) y un $r_{i j} \leq 15 \%$ ), pero se reconoce que de modificar la restricción establecida al coeficiente $r_{i j}$ si contaría con un número de arcos suficientes que le permitirían encajar en tal segmento, dado que, por ejemplo, con un $r_{i j} \leq 20 \%$ su cantidad de arcos sobrepasa la media, por tanto se puede aceptar, una vez eliminada la restricción establecida, que ella es desarrollada bajo el contexto de estudio, aunque lo es en menor grado respecto al primer grupo que se toma como referencia. Tomar tal postura significa asumir que para que se altere en, por ejemplo, un $1 \%$ la producción, la etapa productiva a modificar lo debe hacer como máximo en un $20 \%$, situación que podría implicar un alto costo, dado que queda sujeta a la sensibilidad de la etapa en cuestión.

En lo referente a la detección y posicionamiento del nivel de desarrollo para cada región, se observan diferencias importantes luego de comparar un esquema tradicional como el PIB per cápita por región y uno bajo un contexto input-output. Sobre la base de que las regiones que más PIB per cápita acusan un mayor desarrollo (Tabla 1), se observa que existe una dicotomía entre ambos enfoques: mientras, por ejemplo, en el caso de la Región Metropolitana se lee en la Tabla 1 que tal relación la posiciona en un tercer lugar, bajo el contexto io no sería desarrollada (Anexos 2 y 3), lo que se debería a que no presenta un entramado profuso ni una alta división del trabajo; por otra parte, en regiones como la de Los Lagos, bajo el esquema io se detecta una estructura muy entrelazada, pero basados en su PIB per cápita ella ocupa el décimo lugar; sólo en la región de Atacama se observa una similitud de criterios que nos indican que ella es desarrollada.

En referencia a las diferencias en las respuestas se puede argumentar que ello tiene que ver con la forma en que se obtiene el PIB, recordando que el mismo se puede obtener por la suma simple de los inputs primarios de cada producto o servicio; se da que en el mismo se recogen los excedentes brutos de explotación, los que en algunos casos pueden sobrepasar con creces -en términos de proporción- la producción total, por tanto, lo que allí se mide es más bien renta por los factores productivos y no la forma en que los mismos se elaboran, aspecto que es considerado en el enfoque io por 
medio de sus funciones de producción y distribución, las cuales en este caso quedan supeditadas a elasticidades de sus distintas etapas productivas. Por tanto, se es de la opinión que tal situación explicaría en parte las diferencias detectadas.

\section{TABLA 1}

PIB PER CAPITA POR REGIONES EN MILLONES DE PESOS DE $1996^{8}$

\begin{tabular}{|clrrrc|}
\hline & Región de: & \multicolumn{1}{c}{ PIB } & Población & $\begin{array}{c}\text { PIB/Cápita } \\
(1 \text { US\$ }=\$ 450,80)\end{array}$ & $\begin{array}{c}\text { Ubicación en el } \\
\text { PIB/Cápita }\end{array}$ \\
\hline 1 & Tarapacá & 905.701 & 393.337 & $5.107,83$ & 5 \\
2 & Antofagasta & 1.944 .622 & 468.792 & $9.201,76$ & 1 \\
3 & Atacama & 586.020 & 248.154 & $5.238,50$ & 4 (io-D) \\
4 & Coquimbo & 596.301 & 570.666 & $2.317,93$ & 11 \\
5 & Valparaíso & 2.658 .598 & 1.476 .028 & $3.995,53$ & 6 (io-D) \\
6 & O'Higgins & 1.188 .356 & 762.340 & $3.457,91$ & 8 (io-D) \\
7 & Maule & 1.032 .923 & 989.963 & $2.314,54$ & 12 \\
8 & Biobío & 2.780 .195 & 1.848 .777 & $3.335,85$ & 9 \\
9 & La Araucanía & 773.850 & 850.106 & $2.019,30$ & 13 \\
10 & Los Lagos & 1.249 .677 & 1.047 .227 & $2.647,12$ & 10 (io-D) \\
11 & Aysén & 141.716 & 88.642 & $3.546,46$ & 7 \\
12 & Magallanes & 401.360 & 146.267 & $6.087,01$ & 2 \\
RM & R. Metropolitana & 13.945 .724 & 5.796 .305 & $5.337,11$ & 3 \\
\hline & Total & 28.205 .043 & 14.686 .604 & $4.260,12$ & \\
\hline
\end{tabular}

Fuente: Propia en base a Banco Central e INE.

\section{ACTIVIDADES ECONOMICAS QUE MAS DESTACAN POR IMPULSAR Y CARACTERIZAR A LAS DISTINTAS REGIONES}

Bajo esta clasificación, se observa que los productos agrícolas son importantes en zonas que se caracterizan y prestan para su obtención, a saber, desde la región de O’Higgins a la de Los Lagos (el sur de Chile). Lo mismo ocurre con los productos frutícolas en O'Higgins y Maule (ver Anexos 1, 2 y 3).

Bajo un prisma algo más amplio, el ganado, productos pecuarios y carne son importantes desde la región de Valparaíso (incluyendo la Metropolitana) hasta el sur-austral de Chile, siendo enclave o independiente en el resto del país.

Otro producto que llama la atención es el cobre, que resultó ser clasificado como impulsor de la economía desde la región de Atacama hasta la de O’Higgins, coincidiendo con las zonas donde existen minas cupríferas y señalando que en el sur tal actividad no prevalece. Situación similar se observa con la producción de

8 La expresión (io-D) indica que bajo el esquema io tal región debiera ser considerada desarrollada. 
otros minerales, ellos son importantes en Antofagasta, Atacama, Coquimbo, Aysén y Magallanes. Esto ocurre dado que, en general, para el caso de la minería del cobre existen muchos derivados de ella una vez que el mismo ha pasado por un proceso de fundición, y en el caso del sur-austral, debido a la extracción de minerales tales como el oro, plata y carbón.

Por su parte, las conservas de frutas y vegetales son impulsoras de la economía en casi todo el centro-sur del país (desde la región de Coquimbo hasta la de Aysén, sin incluir a la Metropolitana).

También se observa que los productos derivados de la molinería, pan, fideos y pastas son muy importantes en todo el país, excepto en la región del Biobío.

Finalmente, los productos de la construcción, servicios comerciales, servicios de hotelería y restaurantes y en especial los servicios empresariales, son importantes en todo el país, pues o son clave o son impulsores de la economía.

\section{SIMILITUDES ESTRUCTURALES}

Tipificada la información, se construye una matriz de distancias entre regiones. Así se indaga en primera instancia sobre los parecidos y diferencias que se dan. Esta matriz es cuadrada y simétrica y, en su diagonal principal, todos los elementos son nulos, dado que no existe diferencia para una región consigo misma (Anexo 4).

La medida de distancia empleada es la euclídea al cuadrado $\left[d(i ; j)^{2}=\Sigma_{k}\left(x_{i k}-x_{j k}\right)^{2}\right]$. De la aplicación de esta medida de divergencia, ${ }^{9}$ se puede apreciar que, en orden de importancia, los mayores parecidos se dan para las regiones de Tarapacá y Antofagasta, luego le siguen, respectivamente, Antofagasta con Atacama y Biobío con la región de Los Lagos. Por otra parte, se observa que existe una similitud entre las regiones de Antofagasta con Coquimbo. También presentan cierta semejanza las regiones de Antofagasta y Magallanes y, en el extremo del país, se da cierta analogía entre las regiones de Aysén con Magallanes. En base a estos resultados se puede concluir que existen algunas semblanzas en términos de estructuras productivas para las regiones que conforman la economía chilena.

En segundo lugar, se revisan con mayor profundidad las similitudes estructurales, a partir de los CdI que se forman y se agrupan empleando un clúster jerárquico, dado que permite seguir la "evolución de la agrupación", desde un solo grupo a tantos grupos como elementos existan. Para formar los grupos se consideran las distintas regiones como casos y los CdI como variables (Anexos 2 y 3).

9 Cuando se determina el análisis de clúster en base a la técnica de Ward -procedimiento que tiende a combinar los conglomerados con un número reducido de observaciones-, se recomienda utilizar la distancia euclídea al cuadrado (Hair et al., 2000, pp. 504 y 513). Sin perjuicio de ello, se realizó el mismo análisis empleando la técnica que la intuición señalaba (medidas de correlación), ya que para este caso, lo que interesa es observar los patrones que toman los valores y no sus magnitudes. De este ejercicio, se observó que no existieron diferencias entre ambos procedimientos, con lo cual se aceptan ambos como válidos. Además, se corroboraron los resultados anteriores clusterizando mediante el uso de las $k$-means (clúster no jerárquico), no encontrándose diferencias significativas en las respuestas (ver Anexo 7). 
Del análisis de clúster jerárquico se obtienen siete grupos (Cuadro 1). El primero compuesto por las regiones de Tarapacá, Antofagasta, Atacama y Coquimbo; el segundo, formado por Aysén y Magallanes, etc. Estos resultados pueden recogerse en forma simultánea del dendrograma, que muestra las agrupaciones que pueden constituirse (Anexos 5 y 6 ).

Del análisis de clúster (Anexos 5 y 6), se observa que entre las regiones de Tarapacá y Magallanes existe cierto parecido debido al vínculo que genera el comercio con actividades ligadas al turismo (transporte, hoteles, comunicaciones, etc.). Sin embargo, en Magallanes el comercio está más afianzado, producto de la relación que se da entre éste con otras actividades que no son tan propias del sector turismo (agricultura, frutas, ganado, conservas, aceites y grasas, molinería, alimentos para animales y servicios de esparcimiento y otros).

\section{CUADRO 1}

CLASIFICACION DE LAS REGIONES ANALIZADAS MEDIANTE CLUSTER JERARQUICO

\begin{tabular}{|cl|}
\hline Grupos formados & \multicolumn{1}{c|}{ Región de: } \\
\hline Grupo 1 & Tarapacá, Antofagasta, Atacama y Coquimbo \\
Grupo 2 & Aysén y Magallanes \\
Grupo 3 & Región Metropolitana \\
Grupo 4 & O’Higgins y Maule \\
Grupo 5 & Valparaíso \\
Grupo 6 & Biobío y de Los Lagos \\
Grupo 7 & La Araucanía \\
\hline
\end{tabular}

Fuente: Propia en base a resultados de los Anexos 5 y 6 .

Otro punto interesante que se observa es el que tiene relación con las regiones que presentan ciertas actividades relevantes y que se vinculan al turismo, con estructuras similares y dependientes de lo que se podría denominar "sector turismo". Si bien tal servicio no existe en forma explícita dentro de los 40 productos y actividades considerados, hay algunos de ellos que se podrían vincular a él, por ejemplo, el comercio (en especial en regiones donde hay zonas francas, producto de la llegada de personas que compran allí), la hotelería y restaurantes, los ligados al transporte y, en cierta medida, a las comunicaciones.

Regiones como las de Antofagasta, Atacama, Valparaíso y Metropolitana muestran una clara manifestación de tendencia hacia la especialización del turismo, pues las actividades mencionadas no sólo influyen sobre el resto del sistema económico, sino que, además, sus estructuras, en términos de lo que se ha denominado actividades propias del turismo, son parecidas. 
En regiones como las de Coquimbo, O'Higgins, Maule, La Araucanía, Los Lagos y Aysén, si bien es cierto se desarrolla el turismo, no es muy relevante en términos de integración. Esto es realmente anecdótico, pues en regiones como las de Coquimbo, La Araucanía y Los Lagos, que se caracterizan precisamente por contar con esta actividad, ella no es tan importante como las otras que se desarrollan en su interior y que además guardan más relación con la geografía de estas regiones. En este último sentido, se puede afirmar que si bien el turismo es una actividad importante, su relevancia se ve disminuida cuando es comparada con otras que no sólo están más afianzadas y articuladas, sino que son generadas por las características geográficas propias de cada una de ellas, por ejemplo, la agricultura.

Adicionalmente, del ejercicio formulado, llama la atención lo que se observa en la Región de Los Lagos. Lo planteado obedece a que esta región es reconocida por su alta actividad en silvicultura y agricultura, lo que queda de manifiesto con el ejercicio realizado, pues el ganado, productos pecuarios y de carne se encuentran vinculados a los de productos agrícolas, lácteos y finalmente con la de alimentos para animales, sumado a su propio autoconsumo, es decir, se observa claramente una relación entre productos y actividades propios de los antes mencionados. Por otra parte, se aprecia un segundo grupo dependiente de alimentos para animales, el que por su parte se relaciona con ganado, productos pecuarios y carne, con pescados y mariscos, con aceites y grasas, con productos alimenticios y con otros productos manufacturados. Estos resultados también señalan que para lograr una mayor articulación entre actividades en tal región se requiere que se modifiquen tales productos y actividades en forma mínima. Por ejemplo, en el caso del ganado, productos pecuarios y carne, se deben modificar en un $2,85 \%$ el coeficientes $a_{1 ; 3}$ (relación de compra y venta entre agricultura y ganado) con el fin de aumentar en un $1 \%$ la producción sectorial, lo que traerá como consecuencia un incremento global de la economía mayor. ${ }^{10}$

\section{ANALISIS CAUSAL}

Los bloques no descomponibles corresponderían a clústers que se forman al interior de cada región, partiendo de la base de que existe una estructura totalmente jerarquizada, en cadena causal o recursiva.

10 Elasticidad obtenida a partir de la formulación de Schintke y Stäglin (1988). 


\section{CUADRO 2}

\section{SECTORES NO DESCOMPONIBLES SEGUN REGION}

\begin{tabular}{|ll|}
\hline Región & Productos o actividades motores de la economía \\
\hline Tarapacá & - \\
Antofagasta & - \\
Atacama & $(5-28$ y 30$)$ y $(7$ y 35$)$ \\
Coquimbo & $(1-28$ y 30$)$ \\
Valparaíso & $(2$ y 19$) ;(5-28$ y 30$)$ y $(3$ y 14$)$ \\
O'Higgins & - \\
Maule & - \\
Biobío & $(19-28-30)$ y 36 \\
La Araucanía & $(1-28$ y 30$)$ \\
Los Lagos & $(3-5$ y 14$)$ y 15 \\
Aysén & $(1-28$ y 35$)$ y $(30$ y 40$)$ \\
Magallanes & $(1-6-19-28$ y 38$)$ y $(8$ y 25$)$ \\
Región Metropolitana & $(1-35$ y 40$)$ y $(28$ y 38$)$ \\
\hline
\end{tabular}

Fuente: Elaboración propia.

El Cuadro 2 muestra que no todas las regiones forman bloques no descomponibles (e.g., en Tarapacá, Antofagasta, O’Higgins y Maule), llamando la atención el caso de Antofagasta, región donde se encuentra la mina de cobre a tajo abierto más grande del mundo y se concentra la mayor actividad minera del país, de la que dependen la mayoría de las políticas económicas de Chile, no tiene al menos un clúster minero. Esto indicaría lo poco relevante en términos de integración que es esta actividad en tal zona. En este sentido, se puede aceptar que los commodities, como en este caso el cobre, si no se integran, difícilmente ayudarían al desarrollo de una economía.

La región con el mayor bloque no descomponible (cinco productos o actividades vinculadas $(1,6,19,28$ y 38)) se da en Magallanes (Cuadro 2), luego le sigue Biobío con cuatro (19, 28, 30 y 36) y en tercer lugar, Atacama, Coquimbo, La Araucanía, Los Lagos, Aysén y la Región Metropolitana con tres cada una, respectivamente.

En lo particular, otro grupo que llama la atención es el de frutas, servicios comerciales y transporte, más el que se forma por las actividades agricultura y servicios empresariales en la Región Metropolitana; estos pares de clústers forman lo que se podría denominar actividades propias de la agricultura.

De igual manera, llama la atención lo repetido que están ciertas actividades, e.g., agricultura, comercio y transporte, se repiten en seis de las nueve regiones que presentan bloques (Coquimbo, Biobío, La Araucanía, Aysén, Magallanes y Metropolitana), indicando lo importante que son, entre otros, los productos emanados de la agricultura para las economías regionales de Chile.

En cuanto a los parecidos entre regiones, siguiendo esta técnica, se observa que existen ciertos bloques en común, por ejemplo, Coquimbo con La Araucanía (5, 28 y 30) y en parte con Aysén (1, 28 y 35), similar semejanza se da con un bloque en común para Atacama y Valparaíso (5, 28 y 30). Como se puede apreciar, entre algunas 
regiones existen bloques no descomponibles que acusarían ciertos "parecidos", esto es, la cohesión de las estructuras internas de tales economías obedecería a un número y forma de proceder en sus actividades que desde el punto de vista de sus funciones de producción y cohesión son parecidas.

\section{CONCLUSIONES Y CONSECUENCIAS POLITICAS}

La semejanza y clasificación obtenida para cada región responde al nivel de articulación y vínculo que tienen las diversas actividades en su interior y a las distintas especializaciones por las que cada una es conocida, lo que obedece, en general, a las zonas geográficas de Chile.

Las regiones que más se parecen son la de Tarapacá con Antofagasta, y la del Biobío con Los Lagos, grupos a partir de los cuales puede establecerse el resto de las semejanzas.

Se detectaron dos grandes grupos: uno compuesto por las regiones de Tarapacá, Antofagasta, Atacama, Coquimbo, Aysén y Magallanes, y otro por las regiones de O’Higgins, Maule, Valparaíso, Biobío, Los Lagos y La Araucanía. De estos, el que más se parece a la estructura de la Región Metropolitana es el primero, indicando cuán distinto es el entramado económico de esta región respecto al resto del país.

Las hipótesis planteadas son confirmadas: regiones desarrolladas como las de Valparaíso, Rancagua y Maule o Biobío y Los Lagos presentan estructuras productivas similares. Regiones con actividades menos entrelazas y especializadas, las que acusarían menor desarrollo, presentarían una estructura productiva muy disímil entre ellas y versus las desarrolladas, por ejemplo, la de Aysén versus la de Valparaíso. Esto estaría en línea con lo sostenido por Leontief (1963), Carter (1970), Forsell (1988), Aroche (1996) y Cardero y Aroche (2008).

Por otra parte, los resultados indican que no existe una correlación entre el PIB per cápita y el nivel de entrelazado de las actividades productivas para el nivel de restricciones establecidas, básicamente un $r_{i j} \leq 15 \%$ y un número de arcos igual o mayor que 31 , los que acusarían un mayor nivel de desarrollo bajo un contexto io en la medida que más de estos se tenga, esto es, existe una divergencia importante entre uno de los indicadores tradicionales de desarrollo (un alto PIB per cápita) y el concepto tradicional de desarrollo del mismo pero bajo el esquema aquí tratado (al menos para el caso de la Región Metropolitana).

Desde otra perspectiva, los resultados ayudan a comprender por qué existe más desarrollo en algunas regiones que en otras. Este estaría asociado, según se desprende de los grafos obtenidos, al fruto de múltiples actividades, esto es, a una alta división del trabajo que lleva a una mayor articulación entre productos y servicios.

Actividades características del turismo (hostelería, restauración, transportes y servicios de ocio, deportivos y culturales) no forman un grupo ni siquiera en las regiones donde su presencia es significativa, Valparaíso y Los Lagos. Ello se explica por dos razones: la relativa sencillez de las estructuras que derivan de tal servicio, lo que conlleva una menor capacidad de arrastre, y porque el impacto del turismo es menor del que se suele pensar, aun en las regiones más especializadas. 
Por otra parte, se aprecia que en no todas las regiones se forman grupos no descomponibles (e.g., en Tarapacá, Antofagasta, O’Higgins y Maule), siendo el caso de Antofagasta el que más llama la atención, indicando lo poco relevante en términos de integración y por lo tanto para el desarrollo de ciertas actividades que se generan en las distintas zonas, las cuales marcan las principales actividades que en ellas se realizan. Esto se debería a que, en general, tales regiones se caracterizan por presentar actividades muy básicas (commodities).

De igual forma, la metodología utilizada permite obtener una visión pormenorizada de las regiones estudiadas; ella tiene la particularidad de mostrar desde otro ángulo el efecto que tienen las diferentes elasticidades de las etapas que forman las funciones de producción, a partir de las cuales se van formando los CdI, favoreciendo la visión global del analista, ya que ahora cuenta con una herramienta que le permite indagar no sólo sobre el impacto que tienen la oferta y demanda de insumos, sino que además a cuantos productos o actividades afecta, esto es, se tiene una visión directa e indirecta de los vínculos que posee cada sector con su entorno.

De las políticas públicas a proponer -cuyos fines pueden ser reducir la pobreza, aumentar la producción en aquellas actividades que tiene bajos intercambios o que sean potencialmente generadoras de empleo- se recomienda, a partir de los resultados obtenidos, que:

1. Se promocione a las regiones que poseen atractivos turísticos, dado que no se vislumbran con claridad grupos vinculados a las actividades propias del sector y considerando que las regiones conocidas como "turísticas" (Valparaíso, Coquimbo, Los Lagos, etc.) no forman lo que se podría denominar "clústers turísticos". Una forma de potenciar esto es partiendo por regiones que cuentan con condiciones naturales y, a partir de allí, crear las bases para la formación de clústers ligados al turismo.

2. Hacer más expedito y motivador el acceso a los atractivos naturales. Una mejora puede ser el potenciar las comunicaciones -mejores y más variados accesos- y en aeropuertos - principal vía de ingreso para turistas extranjeros- establecer una imagen de estos en función de los atractivos que presente el lugar, con la finalidad de lograr que dichos recintos cuenten con una identidad propia, que sea una clara manifestación de la identidad de la zona a la cual se arriba. Por ejemplo, para Los Lagos una alternativa puede ser diseñar una fachada que sugiera el ingreso a una región en la cual llueve mucho y que, dada esta condición climática junto a su ubicación y características geográficas, sus casas cuentan con techumbres de tejas de alerce, sin por ello perder de vista los aspectos tecnológicos.

3. Sobre la base de regiones que presentan similitudes estructurales y que cuenten con un grupo de actividades en común, validar éstos a través de grupos de interacción; esto es, reunir a los representantes de aquellos bienes y servicios que forman parte del conjunto y potenciar su unión. De esta forma las políticas públicas que se implementen tendrán como base un grupo de personas vinculadas a determinados productos o actividades y en variadas regiones, lo cual favorecerá no sólo la validación del conjunto, sino que también los objetivos que de él emanen. 
4. Dado que el comercio es la actividad que más interacción mostró, se propone facilitarlo, por ejemplo, apoyando la comercialización de determinados productos; en especial los agrícolas, dada su condición natural de ser productos base. Otra alternativa, para el caso de las regiones extremas en donde existan zonas francas, es permitir liberar automáticamente autos con una cierta data, a condición de que sea uno por persona el que se pueda adquirir, con la finalidad de atraer más turistas y menos comerciantes. Tal propuesta podría significar un mayor ingreso de personas que llegan a las citadas regiones. De esta forma no sólo se incrementaría el flujo de transporte, sino que, además, aumentaría la pernoctación promedio, con las consecuencias propias, que ello traería en actividades tales como las de los restaurantes y hoteles.

5. Al no detectarse un intercambio importante de bienes y servicios entre actividades vinculadas a un proceso en común, una alternativa para su desarrollo sería la creación grupos de trabajo con claras orientaciones tecnológicas e innovadoras. Como resultado se esperaría, por ejemplo, el nacimiento de un clúster basado en la producción del cobre, cuyo fin no sólo sea la búsqueda de una mayor diversificación en su producción y uso, sino que también velar por la factibilidad de los nuevos usos que a este metal se le puedan dar. La idea de tal propuesta es hacer que Chile pase de ser un país exportador de cobre a uno que, en primera instancia, lo extrae y, en segunda, lo manufactura.

6. Potenciar productos y actividades ligados a procesos básicos (agricultura, silvicultura o pesca), pues indirectamente favorecen a comunidades rurales pobres. Se puede repotenciar el apoyo a ellos por medio de la conformación de sociedades; establecidas éstas, el paso siguiente puede ser la ejecución de cursos, en donde se den las herramientas que les permitan una mejor negociación. La ventaja de lo anterior es que se fortalecería la capacidad negociadora de una población que, en general, es carente de educación.

7. Apoyar la generación de empleos en actividades que presentan un alto número de trabajadores, efecto multiplicador o en donde la relación de empleados por unidad de producto sea importante en términos de empleos generados y, además, sus efectos estén distribuidos.

8. Incentivar las actividades que entregan altos encadenamientos y salarios y, a su vez, baja conmutación. Sumado a ello, considerar que, en general, sus CdI formen una gran envergadura, que sean muy profusos. Un ejemplo de lo planteado puede ser para el caso de Magallanes apoyar la molinería, pan y fideos, pues presenta altos encadenamientos y variadas relaciones en sus CdI, y no presenta un éxodo de sus salarios (efecto conmutación); dado que es una actividad que puede hacer simbiosis con el turismo y se puede asociar a empresas familiares pequeñas, tiene un impacto alto y directo en la población local.

\section{DISCUSION Y CAMPOS ABIERTOS}

En referencia a la información utilizada, no hay que olvidar dos aspectos: 1) no existen tablas regionales más actualizadas. Baste recordar que el Banco Central de 
Chile publica sus TIOs Nacionales cada 10 años y sólo ahora ha disminuido ese periodo (1977; 1986; 1996 y 2003), lo que de alguna manera otorga cierta flexibilidad en el uso de los datos empleados; 2) hay estudios que indican que el hacer uso de estos datos no es errado (i.e., Aroca y Atienza, 2008), pues existe una cierta estabilidad en los coeficientes técnicos, lo que permite validar los comentarios relativos a los resultados aquí presentados.

Si bien es cierto se deja de lado la evaluación de encadenamientos (i.e., Rasmussen (1956), Dietzenbacher y van der Linden (1997) o Soza-Amigo (2007), entre otros), se recuerda que el procedimiento utilizado es una alternativa para evaluar la importancia de un producto o actividad. Aun cuando son aspectos y procedimientos distintos, ambas determinan la importancia de una actividad. En este sentido, las respuestas presentadas permiten un mayor acercamiento con la realidad, dado que facilitan la evaluación al presentarse unas respuestas de forma más gráfica, lo que permite una mayor comprensión del fenómeno estudiado.

Los grupos detectados obedecen a un concepto de región basado en criterios geopolíticos que no necesariamente coinciden con los que se puedan identificar luego de considerar estructuras productivas. Este aspecto es importante, porque pueden existir grupos no descomponibles, como el de la leche o el salmón, característicos del sur de Chile, que posiblemente no quedarán totalmente reflejados en el marco geográfico empleado.

Finalmente, se hace necesario incluir en futuros trabajos aspectos tales como la dispersión de compras y ventas en el análisis de los de CdI.

\section{BIBLIOGRAFIA}

AROCA, P. y M. ATIENZA (2008). "La conmutación regional en Chile y su impacto en la Región de Antofagasta”, Revista EURE XXXIV (102), pp. 97-120.

AROCHE, F. (2006). "Sobre los regímenes de crecimiento, el cambio estructural y coeficientes de insumo", El Trimestre Económico LXXIII (4; 292), pp. 881-992.

AROCHE, F. (2005). "Desintegración en la estructura productiva mexicana y el empleo. Los coeficientes importantes y su importancia en la integración”, Revista Asturiana de Economía 32, pp. 31-62.

AROCHE, F. (2002). "Structural transformations and important coefficients in the North American economies", Economic Systems Research 14 (3), pp. 257-273.

AROCHE, F. (1996). "Important coefficients and structural change: A multi-layer approach", Economic Systems Research 8 (3), pp. 235-246.

CARDERO, C. y F. AROCHE (2008). "Cambio estructural comandado por la apertura comercial. El caso de la economía mexicana”, Estudios Económicos 23 (02), pp. 203-252.

CARTER, A. (1970). Structural Change in the American Economy. Harvard University Press.

DIETZENBACHER, E. y J. VAN DER LINDEN (1997). "Sectoral and spatial linkages in the EC production structure", Journal of Regional Science 37 (2), pp. 235-257.

FONTELA, E. y M. GILLI (1977). "The causal structure of economic models", Futures 9 (6), pp. 510-518.

FORSELL, O. (1988). "Growth and changes in the structure of the Finnish economy in the 1960s and 1970s", in: M. CIASCHINI (Ed.), Input-Output Analysis, Chapman and Hall, New York, pp. 287-302.

HAIR, J., R. ANDERSON, R. TATHAM y W. BLACK (1999). Análisis Multivariante. Quinta edición, Madrid, Ed. Prentice Hall, pp. 31-140.

HEWINGS, G., M. FONSECA, J. GUILHOTO y M. SONIS (1989). "Key sectors and structural change in the Brazilian economy: A comparison of alternative approaches and their policy implications", Journal of Policy Modeling 11 (1), pp. 67-90. 
HIRSCHMAN, A. (1958). The strategy of economic development. New Haven, Connecticut, USA, Yale University Press.

HOLUB, H.-W. y H. SCHNABL (1994). Input-output-rechnung: Input-output-analyse. München, Wien: Oldenbourg.

IMBS, J. and R. WACZIARG (2003). "Stages of divertification", The American Economic Journal 93 (1), pp. 63- 86.

LEONTIEF, W. (1963). "The structure of development", Scientific American 209, pp. 148-166.

MILLER, R. and P. BLAIR (2009). "Nonsurvey and partial-survey methods: Fundamentals", in: Inputoutput analysis: Foundations and extensions. Cambridge University Press, pp. 303-346, pp. 750.

MILLER, R. and P. BLAIR (1985). "Nonsurvey and partial-survey methods", in: Input-output analysis: Foundations and extensions. New Jersey, Prentice-Hall Inc., pp. 266-316, pp. 464.

MORILLAS, A. (2005). "Cambios en la estructura productiva española, 1980-1995. Un análisis estructural mediante la Teoría de Grafos", en: TARANCON, M.-A. y M. RAMOS. Estructura input-output y dinámica económica. Editorial Club Universitario (ECU), España, pp. 81-118.

PULIDO, A. y E. FONTELA (1993). Análisis input-output. Modelos, datos y aplicaciones. España. Editorial Pirámide.

RASMUSSEN, P. (1956). Studies in inter-sectoral relations. Amsterdam, North- Holland P. C.

ROBLES, L. y J. SANJUAN (2008). "Sectores y clúster claves en la economía española”, ICE: Tribuna de Economía 843, pp. 183-207.

SCHINTKE, J. y R. STÄGLIN (1988). "Important input coefficients in market transaction tables and production flow tables", in: M. CIASCHINI (Ed.), Input-Output Analysis, Current Developments. Chapman and Hall, New York, pp. 43-60.

SCHNABL, H. (1994). "The evolution of production structures, analyzed by a multi-layer procedure", Economic Systems Research 6 (19), pp. 51-69.

SEBALD, A. (1974). "An analysis of the sensitivity of large scale input-output models to parametric uncertainties", Center for Advanced Computation, document $\mathrm{N}^{\circ} 122$, University of Illinois at Urbana.

SOZA-AMIGO, S. (2009). "Análisis comparativo para la economía magallánica desde la perspectiva de la sensibilidad de coeficientes técnicos", Magallania 37 (1), pp. 133-151.

SOZA-AMIGO, S. (2008). "Análisis comparativo para la economía magallánica desde la perspectiva del enfoque input-output", Revista de Análisis Económico 23 (2), pp. 95-120.

SOZA-AMIGO, S. (2007). "Análisis estructural input-output: Antiguos problemas y nuevas soluciones", Tesis (Doctorado en Economía Aplicada). Universidad de Oviedo, Departamento de Economía Aplicada, España.

WEST, G. (1982). "Sensitivity and key sector analysis in input-output models", Australian Economic Papers 21, pp. 365-378. 


\section{ANEXO 1}

\section{RELACION ENTRE EL NUMERO DE PRODUCTO DE ACTIVIDAD (RAMA) Y LO QUE REPRESENTA}

\begin{tabular}{|c|c|}
\hline $\begin{array}{l}\text { Clasificador/ } \\
\text { Número }\end{array}$ & Clasificador de producto o actividad \\
\hline 1 & Productos agrícolas \\
\hline 2 & Frutas \\
\hline 3 & Ganado; productos pecuarios y carne \\
\hline 4 & Productos silvícolas; maderas y papel \\
\hline 5 & Pescados y mariscos frescos y productos del mar industrializados \\
\hline 6 & Petróleo crudo y combustibles y lubricantes \\
\hline 7 & Cobre \\
\hline 8 & Otros minerales \\
\hline 9 & Conservas de frutas y vegetales \\
\hline 10 & Aceites y grasas \\
\hline 11 & Productos lácteos \\
\hline 12 & Productos de molinería, pan, fideos y pastas \\
\hline 13 & Otros productos alimenticios \\
\hline 14 & Alimentos para animales \\
\hline 15 & Licores; cervezas; bebidas analcohólicas y vinos \\
\hline 16 & Productos del tabaco \\
\hline 17 & Productos textiles, prendas de vestir, cuero y calzado \\
\hline 18 & Impresos y grabaciones \\
\hline 19 & Productos químicos, de caucho y de plástico \\
\hline 20 & Vidrio, productos de vidrio, y productos de minerales no metálicos \\
\hline 21 & Productos básicos de hierro y acero y productos metálicos \\
\hline 22 & Maquinaria y equipo eléctrico y no eléctrico \\
\hline 23 & Muebles \\
\hline 24 & Otros productos manufactureros \\
\hline 25 & Electricidad \\
\hline 26 & Gas y Agua \\
\hline 27 & Productos de la construcción \\
\hline 28 & Servicios comerciales \\
\hline 29 & Servicios de hotelería y de restaurantes \\
\hline 30 & Servicios de transporte ferroviario y caminero \\
\hline 31 & Servicios de transporte marítimo y aéreo \\
\hline 32 & Servicios conexos de transporte \\
\hline 33 & Servicios de comunicaciones \\
\hline 34 & Servicios financieros y de seguros \\
\hline 35 & Servicios empresariales \\
\hline 36 & Servicios inmobiliarios y de propiedad de vivienda \\
\hline 37 & Servicios de administración pública \\
\hline 38 & Servicio de educación pública y privada \\
\hline 39 & Servicio de salud pública y privada \\
\hline 40 & Servicios de esparcimiento y otros servicios \\
\hline
\end{tabular}

Fuente: MIDEPLAN (2005). 


\section{ANEXO 2}

\section{PARA LAS DISTINTAS REGIONES ${ }^{11}$}

Tarapacá (1)

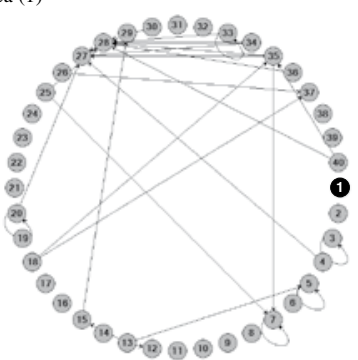

Atacama (3)

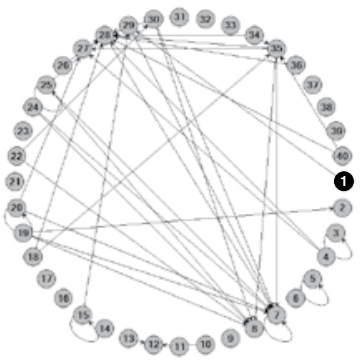

Valparaíso (5)

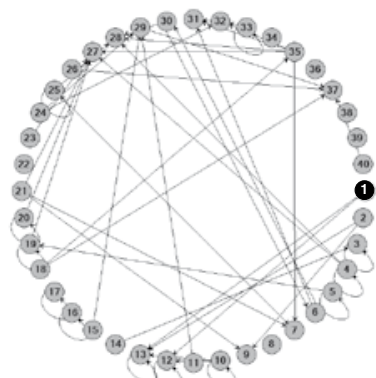

Antofagasta (2)

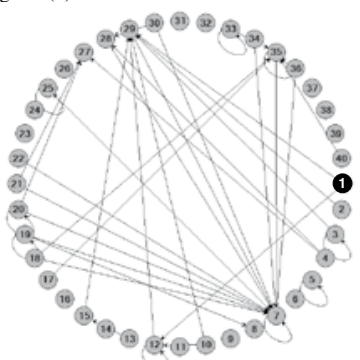

Coquimbo (4)

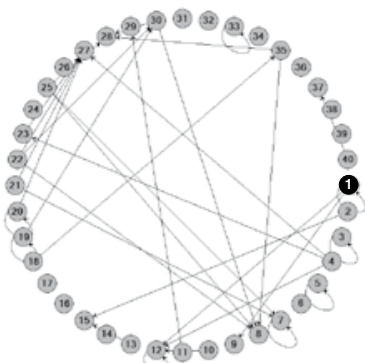

Del Libertador Bernardo O’Higgins (6)

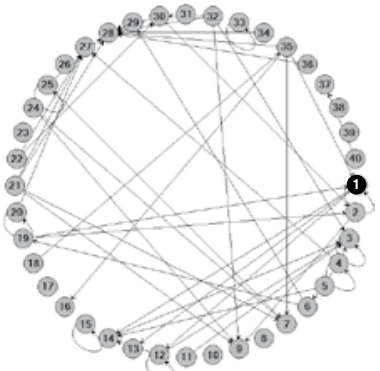

11 Con la finalidad de facilitar la lectura, el primer sector se presenta con otro color. De igual forma, se indica que el orden de las ramas sigue el sentido de un reloj tradicional. 
(continuación Anexo 2)

Maule (7)

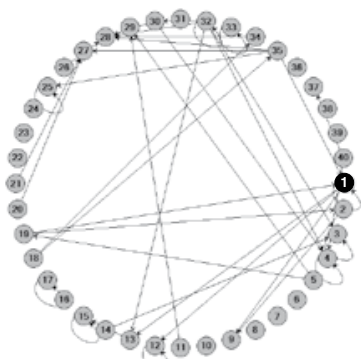

La Araucanía (9)

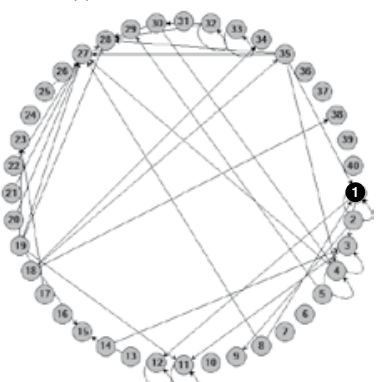

Aysén (11)

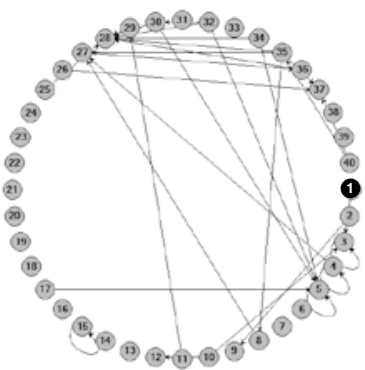

Metropolitana (RM)

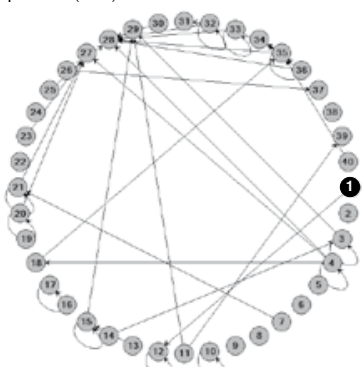

Biobío (8)

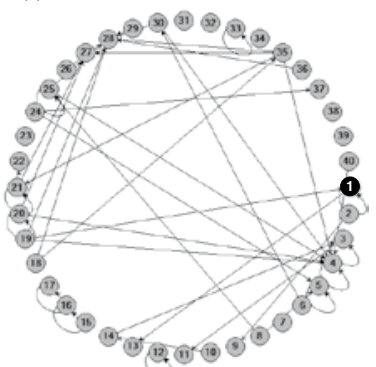

Los Lagos (10)

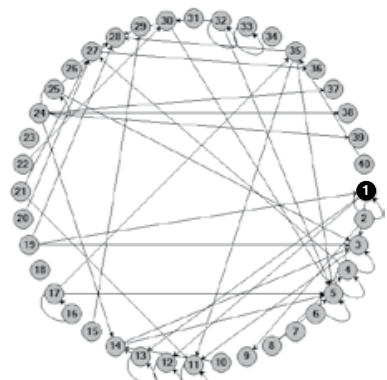

Magallanes (12)

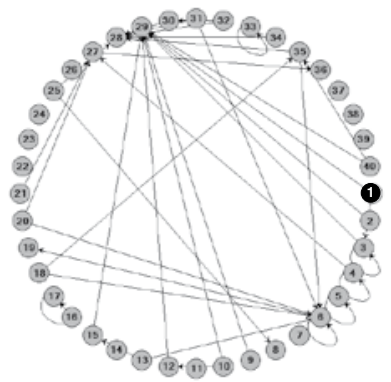

Fuente: Elaboración propia. 


\section{ANEXO 3}

\section{RESUMEN DE ACTIVIDADES SEGUN TIPO DE RELACION QUE POSEE LA RAMA EN FUNCION DE SUS CDI}

\begin{tabular}{|c|c|c|c|c|c|c|c|c|c|c|c|c|c|}
\hline Sector & R1 & $\mathrm{R} 2$ & R3 & R4 & R5 & R6 & R7 & R8 & R9 & R10 & R11 & R12 & RM \\
\hline s1 & E & B & E & B & B & $\mathrm{C}$ & $\mathrm{C}$ & C & $\mathrm{C}$ & $\mathrm{C}$ & E & B & E \\
\hline s2 & E & E & M & E & B & C & $\mathrm{C}$ & E & E & E & E & E & E \\
\hline s3 & E & E & $\mathrm{E}$ & E & M & M & M & $\mathrm{C}$ & $\mathrm{C}$ & C & M & $\mathrm{C}$ & $\mathrm{C}$ \\
\hline s4 & $\mathrm{E}$ & B & B & B & B & B & M & M & C & C & $\mathrm{E}$ & E & B \\
\hline s5 & M & E & E & $\mathrm{E}$ & E & E & B & M & E & M & M & E & E \\
\hline s6 & E & E & E & $\mathrm{E}$ & $\mathrm{E}$ & C & E & $\mathrm{E}$ & E & $\mathrm{E}$ & $\mathrm{E}$ & C & E \\
\hline s7 & M & M & M & M & M & M & E & E & E & E & E & $\mathrm{E}$ & E \\
\hline s8 & E & M & M & M & E & $\mathrm{E}$ & $\mathrm{E}$ & B & B & B & C & M & E \\
\hline s9 & E & E & E & M & M & M & M & M & M & M & M & E & E \\
\hline s10 & E & B & E & $\mathrm{E}$ & B & $\mathrm{E}$ & $\mathrm{E}$ & $\mathrm{E}$ & $\mathrm{E}$ & $\mathrm{E}$ & B & B & E \\
\hline s11 & $\mathrm{E}$ & E & E & $\mathrm{E}$ & $\mathrm{E}$ & E & E & M & M & M & $\mathrm{E}$ & E & B \\
\hline s12 & M & C & M & M & M & C & M & $\mathrm{E}$ & M & M & M & C & M \\
\hline s13 & B & E & $\mathrm{E}$ & $\mathrm{E}$ & M & $\mathrm{M}$ & C & M & E & C & E & B & E \\
\hline s14 & E & E & E & E & $\mathrm{E}$ & C & E & C & E & C & E & E & E \\
\hline s15 & C & C & E & $\mathrm{M}$ & E & E & M & $\mathrm{E}$ & M & E & $\mathrm{E}$ & C & C \\
\hline s16 & E & E & E & $\mathrm{E}$ & E & M & $\mathrm{E}$ & E & $\mathrm{E}$ & E & $\mathrm{E}$ & E & E \\
\hline s17 & E & E & E & $\mathrm{E}$ & E & $\mathrm{E}$ & E & E & E & B & $\mathrm{E}$ & E & $\mathrm{E}$ \\
\hline s18 & B & B & B & $\mathrm{E}$ & B & E & B & B & B & E & E & B & C \\
\hline s19 & $\mathrm{E}$ & B & B & E & C & $\mathrm{C}$ & C & B & B & B & E & M & $\mathrm{E}$ \\
\hline s20 & E & B & B & E & $\mathrm{E}$ & E & E & B & E & E & E & B & $\mathrm{E}$ \\
\hline s21 & E & B & E & B & B & B & E & C & E & B & E & E & C \\
\hline s22 & E & E & B & B & $\mathrm{E}$ & B & E & M & E & E & E & E & E \\
\hline s23 & E & E & E & C & $\mathrm{E}$ & E & E & $\mathrm{E}$ & M & E & E & $\mathrm{E}$ & E \\
\hline s24 & E & E & B & E & B & B & $\mathrm{E}$ & B & $\mathrm{E}$ & B & E & E & E \\
\hline s25 & B & E & B & B & B & B & $\mathrm{M}$ & C & E & B & E & B & E \\
\hline s26 & E & E & E & E & E & E & E & E & E & E & E & E & E \\
\hline s27 & M & M & M & $\mathrm{M}$ & M & M & $\mathrm{M}$ & $\mathrm{M}$ & $\mathrm{M}$ & $\mathrm{C}$ & $\mathrm{C}$ & $\mathrm{C}$ & $\mathrm{M}$ \\
\hline s28 & C & C & C & $\mathrm{M}$ & M & M & $\mathrm{M}$ & $\mathrm{M}$ & $\mathrm{M}$ & $\mathrm{M}$ & M & $\mathrm{M}$ & $\mathrm{M}$ \\
\hline s29 & M & C & C & M & $\mathrm{C}$ & $\mathrm{C}$ & M & $\mathrm{E}$ & M & M & $\mathrm{C}$ & $\mathrm{M}$ & $\mathrm{M}$ \\
\hline s30 & $\mathrm{E}$ & B & C & C & $\mathrm{E}$ & C & C & C & C & C & C & C & $\mathrm{E}$ \\
\hline s31 & E & E & E & $\mathrm{E}$ & $\mathrm{M}$ & $\mathrm{E}$ & E & E & E & E & E & B & $\mathrm{M}$ \\
\hline s32 & E & E & E & E & C & B & B & E & B & B & B & B & B \\
\hline s33 & E & E & E & $\mathrm{E}$ & E & E & E & E & $\mathrm{E}$ & $\mathrm{E}$ & E & E & B \\
\hline s34 & B & E & B & $\mathrm{E}$ & $\mathrm{E}$ & $\mathrm{E}$ & C & E & M & $\mathrm{E}$ & B & E & $\mathrm{M}$ \\
\hline s35 & C & C & $\mathrm{C}$ & $\mathrm{C}$ & $\mathrm{C}$ & $\mathrm{C}$ & C & $\mathrm{C}$ & $\mathrm{C}$ & $\mathrm{C}$ & C & $\mathrm{C}$ & $\mathrm{C}$ \\
\hline s36 & $\mathrm{E}$ & E & E & $\mathrm{E}$ & $\mathrm{E}$ & $\mathrm{E}$ & $\mathrm{E}$ & $\mathrm{E}$ & E & M & C & M & $\mathrm{E}$ \\
\hline s37 & $\mathrm{M}$ & E & E & M & $\mathrm{M}$ & M & M & $\mathrm{M}$ & $\mathrm{E}$ & $\mathrm{M}$ & M & E & $\mathrm{M}$ \\
\hline s38 & E & E & E & $\mathrm{E}$ & $\mathrm{E}$ & $\mathrm{E}$ & $\mathrm{E}$ & $\mathrm{E}$ & $\mathrm{M}$ & $\mathrm{M}$ & $\mathrm{E}$ & E & $\mathrm{E}$ \\
\hline s39 & E & E & E & E & $\mathrm{E}$ & E & E & E & $\mathrm{E}$ & $\mathrm{M}$ & $\mathrm{E}$ & E & $\mathrm{M}$ \\
\hline $\mathrm{s} 40$ & B & E & B & E & $\mathrm{E}$ & E & B & E & $\mathrm{E}$ & $\mathrm{E}$ & B & B & $\mathrm{E}$ \\
\hline
\end{tabular}

Donde: $\mathrm{C}$, indica que es una rama clave; $\mathrm{M}$, impulsora de la economía; $\mathrm{B}$, es un sector que ofrece más que el promedio al resto del sistema productivo; E, señala que es una actividad que no presenta vínculos con otras. Por otro lado, de este resumen se han excluido los autoconsumos. Las R hacen referencia a las regiones, ordenadas de norte a sur (antigua clasificación). Finalmente, RM se refiere a la Región Metropolitana. 


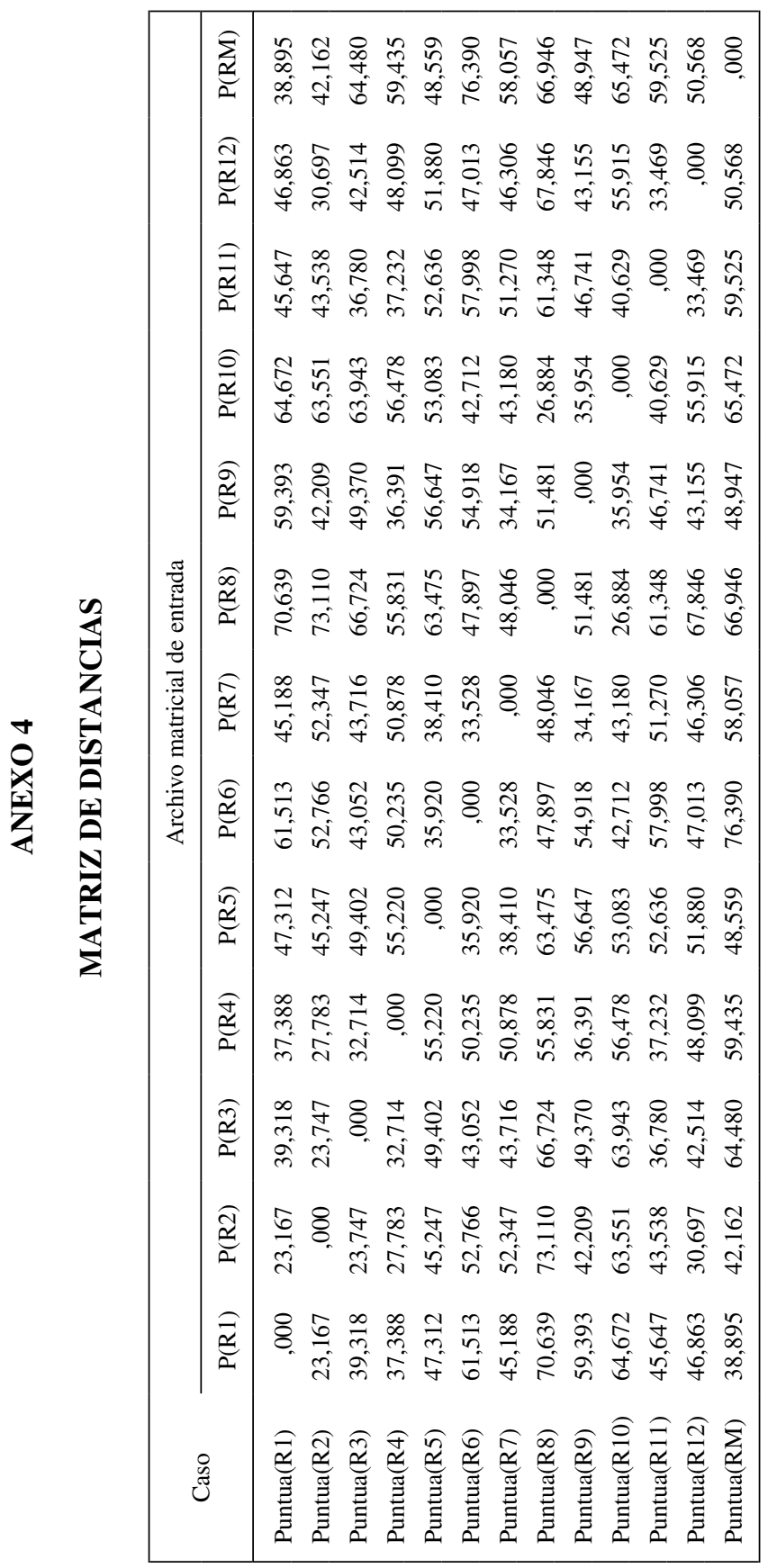




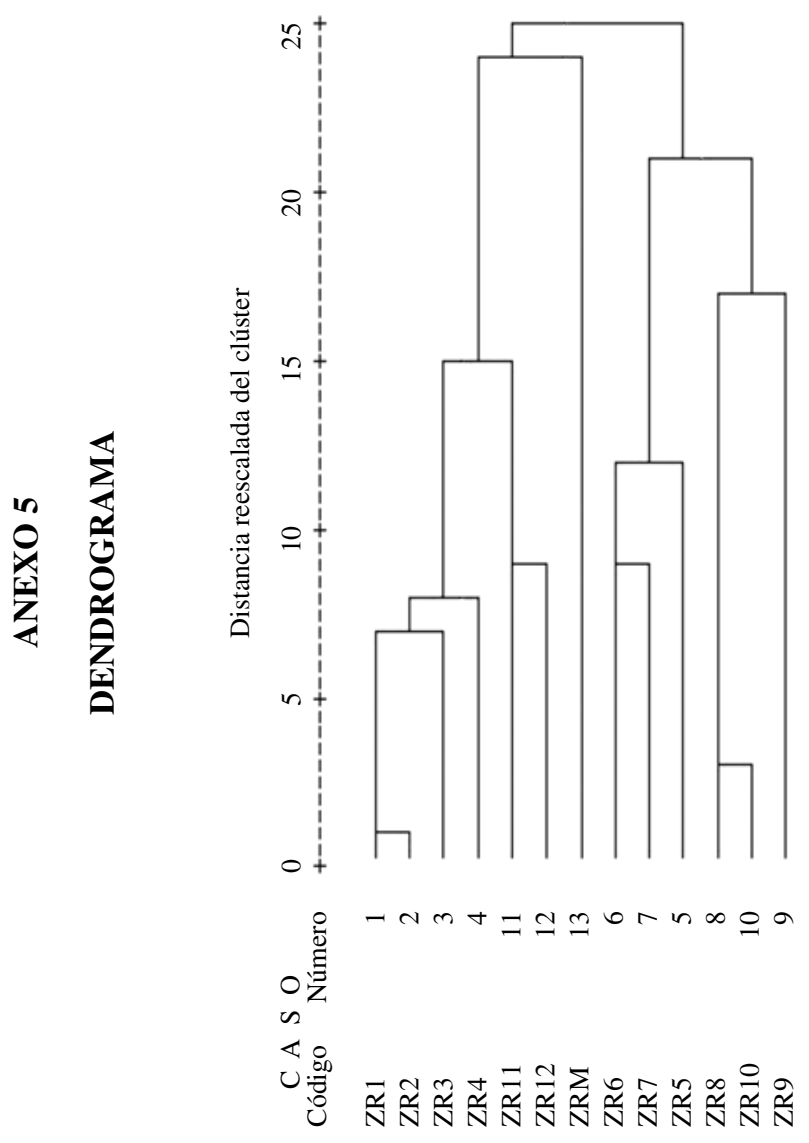




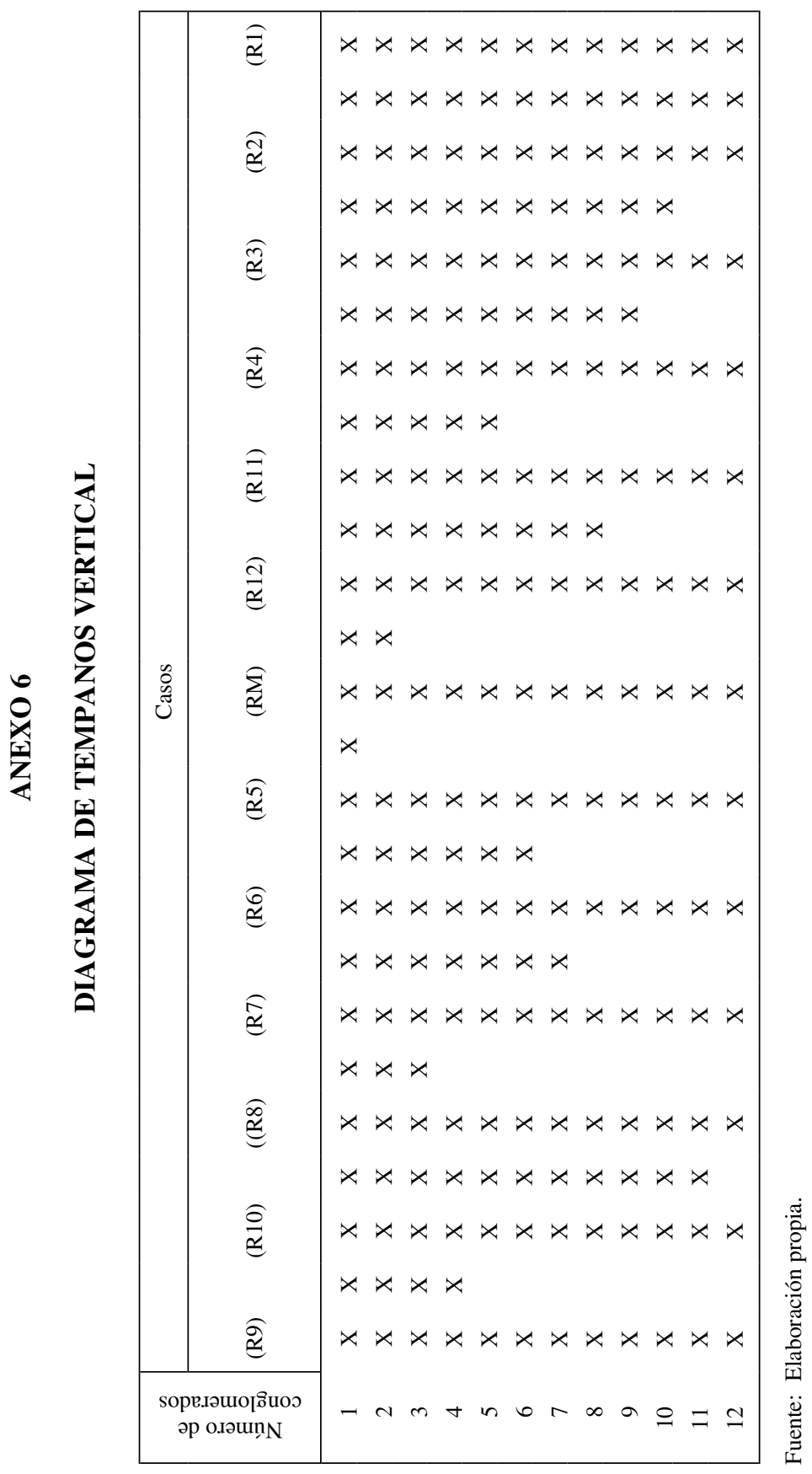




\section{ANEXO 7}

\section{PERTENENCIA A LOS CONGLOMERADOS}

\begin{tabular}{|c|c|c|}
\hline $\begin{array}{c}\text { Número de caso } \\
\text { (Región) }\end{array}$ & Conglomerado & Distancia \\
\hline R1 & 1 & 3,354 \\
R2 & 1 & 2,461 \\
R3 & 1 & 3,382 \\
R4 & 4 &, 000 \\
R5 & 5 &, 000 \\
R6 & 6 & 2,895 \\
R7 & 6 & 2,895 \\
R8 & 8 & 2,592 \\
R9 & 2 &, 000 \\
R10 & 8 & 2,593 \\
R11 & 2,893 \\
R12 & 3 & 2,893 \\
R13 & 3 &, 000 \\
\hline
\end{tabular}

